\title{
Long versus Short Segment Instrumentation in Osteoporotic Thoracolumbar Vertebral Fracture
}

\author{
Massimo Girardo ${ }^{1}$, Alessandro Massè ${ }^{2}$, Salvatore Risitano ${ }^{3}$, Federico Fusini $^{1}$ \\ ${ }^{1}$ Spine Surgery Unit, Orthopaedic and Trauma Centre, Azienda Ospedaliera Città della Salute e della Scienza, Turin, Italy \\ ${ }^{2}$ Department of Orthopaedic and Traumatology, Orthopaedic and Trauma Centre, Azienda Ospedaliera Città della Salute e della Scienza, \\ University of Turin, Turin, Italy \\ ${ }^{3}$ Department of Orthopaedic Surgery and Traumatology, Maggiore Hospital of Chieri, Turin, Italy
}

Study Design: Retrospective comparative study.

Purpose: This study aimed to compare clinical and radiological data and rate of mechanical complications in elderly patients treated with short segment (SSS) or long segment stabilization (LSS) for thoracolumbar junction osteoporotic vertebral fractures (OVFs).

Overview of Literature: A fervent debate is now focused on the treatment of OVF using SSS or LSS. High rate of complications is associated with pedicle screw fixation because of poor bone quality.

Methods: Patients over 65 years old with a T-score of <-2.5, affected by (T10-L2) vertebral fracture treated with LSS or SSS pedicle screw fixation, with at least 24 months of follow-up were evaluated. All patients were analyzed with conventional X-ray to evaluate bisegmental kyphotic angle (BKA) and clinically with Visual Analog Scale (VAS), Oswestry Disability Index (ODI), and rate of mechanical complications at 2, 6, 12, and 24 months. Data were expressed as mean \pm standard deviation. Student $t$-test was used to compare clinical scores between populations. Mann-Whitney $U$-test was used to analyze clinical and radiological variable, whereas Fisher's exact test was used to identify differences in the rate of complications between groups.

Results: A total of 37 patients met the inclusion criteria. Mean follow-up was $33.97 \pm 9.26$ months. For both groups, ODI and VAS significantly decreased over time with good results $(p<0.00001)$. At the final follow-up, no significant differences were found in terms of $\mathrm{ODI}$ and VAS. There was no difference in correction of BKA between groups; however, a significant difference was found in LSS group between pre- and postoperative BKA $(p=0.046)$, whereas no difference was found in SSS group. A significant difference in the rate of mechanical complications was found between groups $(p=0.011)$.

Conclusions: Both treatments showed good clinical and radiological results; however, LSS group showed better BKA correction and lower mechanical complications than SSS group.

Keywords: Fragility vertebral fracture; Long segment fixation; Short segment fixation; Augmentation

\section{Introduction}

Most of the elderly population are affected by osteoporosis, presenting as a major health burden owing to increased risk of fracture and subsequent disability or related death. Low bone density affects bone strength, particularly in the vertebral body $[1,2]$. Surgical treatment for osteoporotic vertebral fracture (OVF) is still challeng-

\footnotetext{
Received Jan 26, 2020; Revised Apr 20, 2020; Accepted May 8, 2020

Corresponding author: Federico Fusini

Orthopaedic and Traumatology, Orthopaedic and Trauma Centre, via zuretti 29, Torino, 10126, Italy

Tel: +39-0116933290, Fax: +39-0116933291, E-mail: fusinif@hotmail.com
} 
ing for spine surgeons. The literature reports several approaches to treatment, but there is no current consensus on a standard technique [3-7]. Compared with younger population, high rate of complications is associated with pedicle screw fixation because of poor bone quality such as loosening, pullout, or screw migration [8].

Improvement of fixation technique and new pedicle screw shape could help spine surgeons in the treatment of challenging cases $[5,9]$. However, despite these features, the risk of treatment failure is still high $[10,11]$. Currently, a fervent debate is focused on the treatment for OVF using short segment (SSS) or long segment stabilization (LSS). Some surgeons prefer to save motion of adjacent segments, hence choosing SSS, whereas other surgeons opt to obtain a more stable construct with LSS but sacrificing some segment of motion $[12,13]$.

This study aimed to evaluate and compare clinical and radiological data and rate of mechanical complications in patients treated with SSS or LSS for OVF of the thoracolumbar junction.

\section{Materials and Methods}

\section{Study design}

Clinical and radiological data of patients treated in our institution using long or short segment pedicle screw fixation for osteoporotic fracture of the thoracolumbar spine (T10-L2) were collected and reviewed. All patients were evaluated using radiological examination with conventional X-ray and clinically with Visual Analog Scale (VAS) and Oswestry Disability Index (ODI) at 2, 6, 12, and 24 months. The need for surgical revision and complications were also recorded.

\section{Selection and description of participants}

Between January 2015 and December 2016, 137 consecutive patients affected by thoracolumbar vertebral fracture were admitted in our Orthopaedic and Trauma Centre, Azienda Ospedaliero Universitaria Città della Salute e della Scienza di Torino, Turin. Their records were identified using a retroactive revision of our database using the TrakCare program (InterSystems Corp., Cambridge, MA, USA).

We included patients over 65 years of age with a T-score of $<-2.5$, affected by T10-L2 vertebral fractures treated in our institution using SSS (one level above and under the fracture with intermediate screws in the affected vertebra) or LSS (two level above and below the affected vertebra, with or without intermediate screws) with or without polymethylmethacrylate (PMMA) augmentation. All patients were affected by osteoporotic fractures $(\mathrm{OF}) 3$, OF 4, or OF 5 types of vertebral fracture according to the German Society for Orthopaedics and Trauma (DGOU) classification [14] and an OF classification-based scoring system $>6$.

The exclusion criteria were as follows: polytrauma, inflammatory diseases such as ankylosing spondylitis and rheumatoid arthritis, history of major surgery of the thoracolumbar spine (i.e., arthrodesis or with a combined approach [posterior+anterior]) or treated with vertebroplasty (VP) and kyphoplasty (KP) of the index vertebra, incomplete radiological or clinical data, or less than 24 months of follow-up.

\section{Treatments}

All patients underwent a posterior stabilization with pedicle screws using an open approach with a freehand technique under general anesthesia. Intermediate shortsegment pedicle screws in the fractured vertebra were positioned according to pedicle integrity. In case of single pedicle fracture, screws were positioned asymmetrically, whereas no screws were positioned in case of bilateral pedicle fracture. Screw positioning was checked intraoperatively with fluoroscopic guidance. After surgery, all patients were free to move without support or corset. Generally, fractures with small fragmentation and comminuted fragments with acceptable kyphosis were treated with SSS (Figs. 1,2) using intermediate pedicle screws according to pedicle integrity. In case of great fragmentation and comminuted fragments and segment kyphosis of $>20^{\circ}$, an LSS was preferred (Fig. 3). Usually, stand-alone VP or KP can be used to successfully treat wedge fractures (AO A1 or $\mathrm{OF} 2$ ) or incomplete burst fractures (AO A3 or OF 3 ) after 3 weeks of unsuccessful conservative treatment because of persistent pain $[15,16]$. In case of major posterior wall displacement or interruption, pedicle screws are preferable instead of VP or KP owing to risk of iatrogenic narrowing of the spinal canal [15].

Solid pedicle screws or augmented cannulated pedicle screws were used in all cases. No cannulated pedicle screws without PMMA augmentation were used, based on 

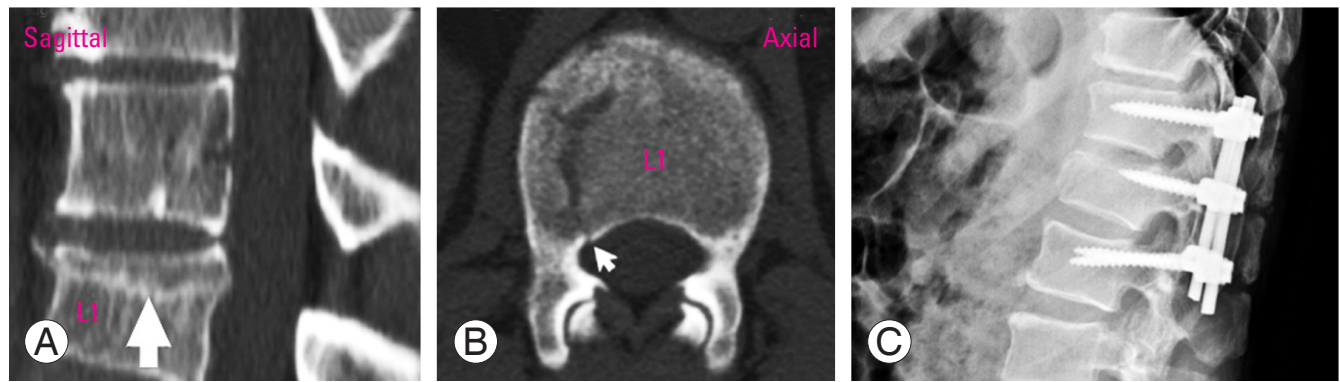

Fig. 1. Preoperative computed tomography scan with evidence of involvement of posterior wall in sagittal (A) and axial view (B) and postoperative L1 vertebral fracture X-ray (C) of a 78-year-old male patient treated with short segment instrumentation with short screw in affected vertebra without augmentation (arrow).
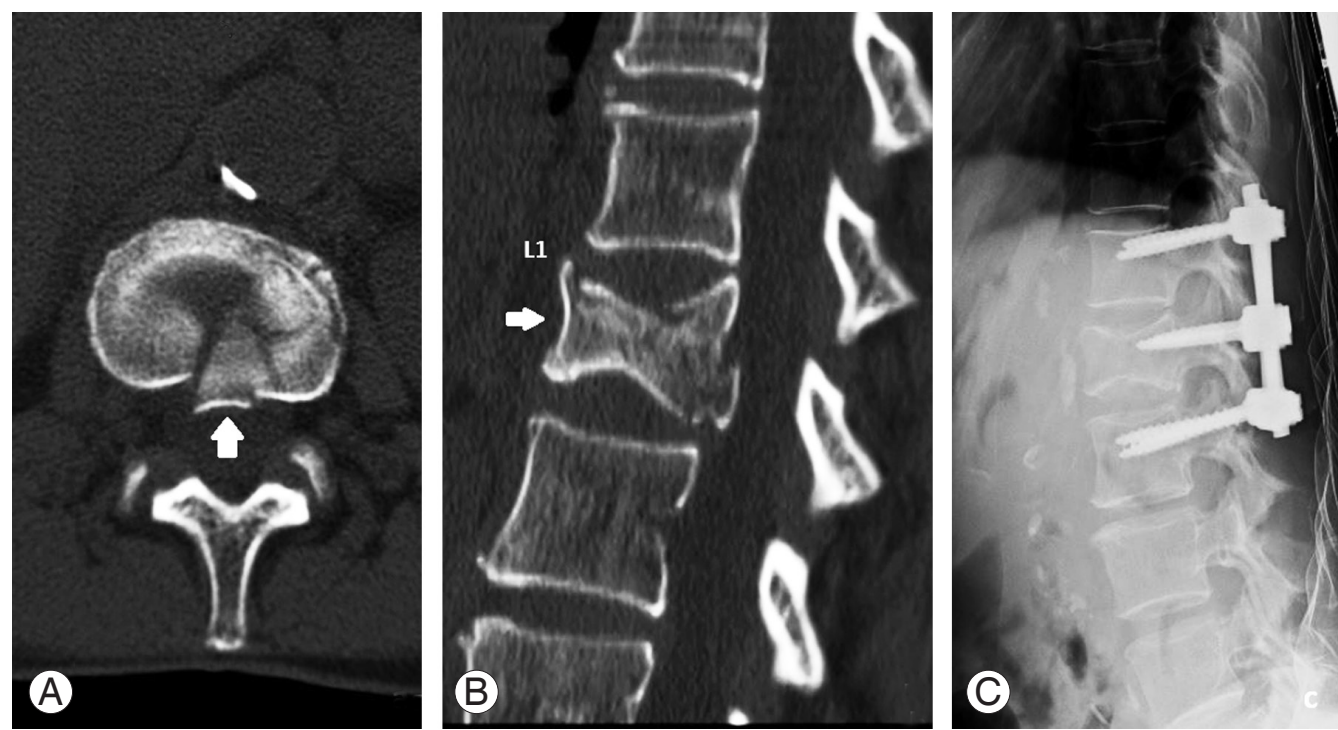

Fig. 2. Preoperative computed tomography scan evaluation in axial (A) and sagittal (B) view of a 76-year-old female patient with L1 vertebral fracture (arrow). (C) Postoperative X-ray of fracture treated with short segment fixation without augmentation.

the results of our previous work [4].

\section{Radiological assessment}

An independent radiologist, not involved in the study, evaluated the radiological data of all patients: thoracolumbar anteroposterior and lateral X-rays at 40 days, and 6, 12, and 24 months. Fractures were classified according to OF classification system [17]. Bisegmental kyphotic angle (BKA) on conventional X-ray was evaluated before treatment and at each follow-up.

\section{Measurements}

During follow-up, patients were clinically evaluated with VAS and ODI. All complications were recorded; proximal junctional kyphosis (PJK), implant loosening, pullout, or screw breakage were all considered as mechanical complications.

The study was conducted according to the Declaration of Helsinki and its later amendments. Ethical approval was obtained from Inter-Hospital Ethical Committee (no., 14/0006482). All patients provide written informed consent prior participation to the study.

\section{Statistical analysis}

Collected data are expressed as mean \pm standard deviation. Student $t$-test was used to compare clinical scores between populations. Mann-Whitney $U$-test was used to analyze clinical and radiological variables, whereas Fisher's exact test was used to identify differences in the rate of complications between short segment group and long segment group or between solid pedicle screws or augmented 

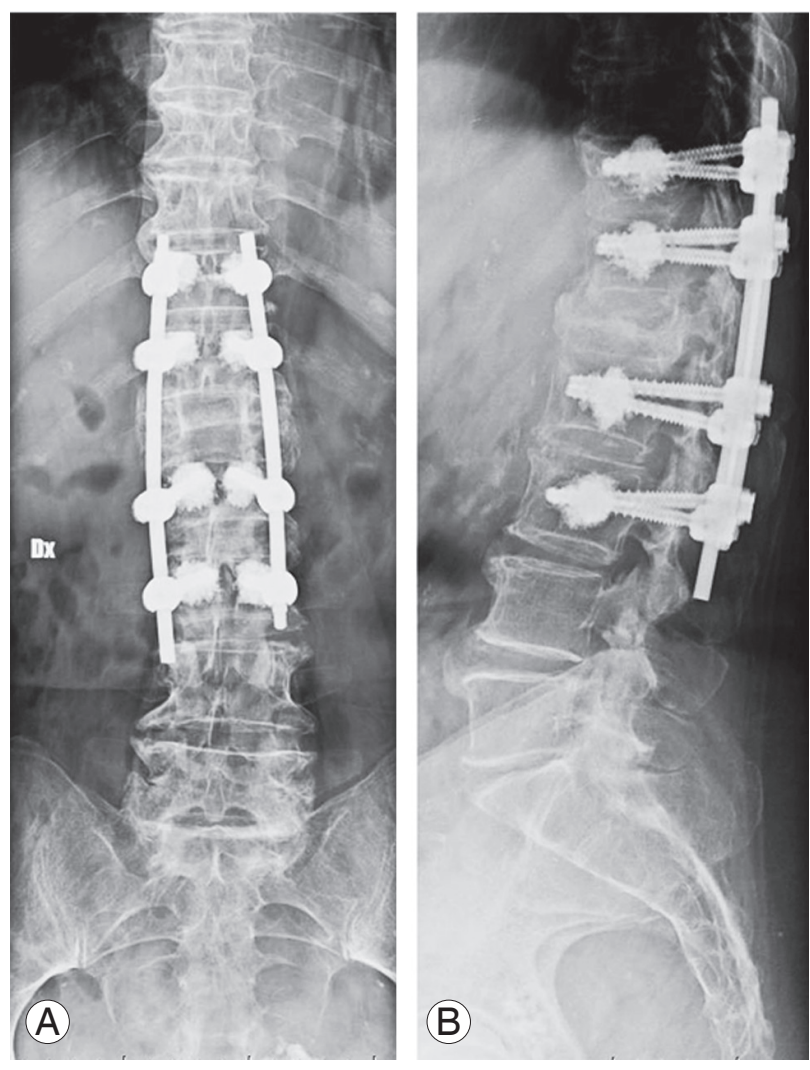

Fig. 3. (A, B) Postoperative $X$-ray in anteroposterior and lateral view of $L 1$ vertebral fracture of a 66 -year-old female patient treated with long segment fixation (two level above and below affected vertebrae) with polymethylmethacrylate without intermediate screw.

cannulated pedicle screws in each group. A $p<0.05$ was considered statistically significant. Stata ver. 13.0 software (Stata Corp., College Station, TX, USA) was used for statistical analysis.

\section{Results}

A total of 37 patients (22 females and 15 males) met the inclusion criteria. Mean follow-up was 33.97 \pm 9.26 months. Demographic data and fracture types are presented in Table 1. Both groups were similar preoperatively.

For both groups, ODI and VAS significantly decreased over time with good results $(p<0.00001)$. At the final follow-up, no significant differences were found in term of ODI ( $p=0.906)$ and VAS ( $p=0.853)$. Statistical analysis showed no difference in correction of BKA between groups ( $p=0.785$ ); however, a significant difference was found he in LSS group between pre- and postoperative BKA $(p=0.046)$, whereas the same difference was not found in the SSS group $(p=0.269)$. All complications are
Table 1. Main demographic characteristics of patients included in the study with OF classification distribution

\begin{tabular}{|c|c|}
\hline Characteristic & Value \\
\hline Mean age (yr) & $70.38 \pm 5.45$ \\
\hline \multicolumn{2}{|l|}{ Gender } \\
\hline Female & 22 \\
\hline Male & 15 \\
\hline \multicolumn{2}{|l|}{ Long segment stabilization group } \\
\hline Male & 7 \\
\hline Female & 15 \\
\hline OF classification & 18 OF4, 4 OF5 \\
\hline \multicolumn{2}{|l|}{ Short segment stabilization group } \\
\hline Male & 8 \\
\hline Female & 7 \\
\hline OF classification & 12 OF3, 3 OF4 \\
\hline Total & 37 \\
\hline OF classification & 12 OF3, 21 OF4, 4 OF5 \\
\hline \multicolumn{2}{|l|}{ Mechanism of injury } \\
\hline No relevant trauma ${ }^{\text {al }}$ & 9 \\
\hline Minor trauma ${ }^{\text {bl }}$ & 15 \\
\hline Fall from height ${ }^{c \mid}$ & 8 \\
\hline Other moderate energy trauma ${ }^{d)}$ & 5 \\
\hline
\end{tabular}

Values are presented as mean \pm standard deviation or number.

OF, osteoporotic fractures.

${ }^{\text {a) }}$ No relevant trauma connected to vertebral injury, i.e., twisting, coughing, sneezing, etc. ${ }^{b}$ Fall from standing, seated position or from bed. ${ }^{\text {cl} F a l l ~ f r o m ~ l e s s ~}$ than $1 \mathrm{~m}$ height. ${ }^{\mathrm{d}} \mathrm{C}$ ar accident at low speed, pedestrian hit at extremely low speed, etc.

Table 2. Complications overview of patients included in the study and treated with SSS or LSS

\begin{tabular}{lcccc} 
Variable & LSS & SSS & $p$-value & Global $p$-value \\
Wound dehiscence & 2 & 2 & 1 & 1 \\
Leakage & 3 & 1 & 0.633 & \\
\hline Pulmonary embolism & 1 & - & 1 & \\
Transitory paresthesia & - & 1 & 0.41 & \\
\hline Proximal junctional kyphosis & 1 & 1 & 1 & 0.011 \\
Loosening & - & 2 & 0.16 & \\
\hline Pull out & - & 3 & 0.059 & \\
\hline
\end{tabular}

SSS, short segment stabilization; LSS, long segment stabilization.

presented in Table 2. One mechanical complication was reported for the LSS group (one PJK), whereas six mechanical complications were reported for the SSS group (three pullout, one PJK, two loosening). A significant difference was found between groups for mechanical com- 
plications $(p=0.011)$.

In the subgroup analysis, 12 patients were treated with solid screws and 10 patients with augmented cannulated pedicle screws in the LSS group, whereas nine patients were treated with solid screws and six with augmented cannulated pedicle screws in the SSS group. Patients with PJK in the LSS group were treated with solid screws, whereas those in the SSS group were treated with cannulated augmented pedicle screws. All patients with other mechanical complications in the SSS group (pullout and screw loosening) were treated with solid screws. No difference was found in global mechanical failure between solid or augmented cannulated pedicle screws $(p=0.11)$. For screw pullout and loosening, no difference was observed in the LSS group $(p=1)$, whereas a significant statistical difference was found in the SSS group $(p<0.044)$.

\section{Discussion}

Choosing between SSS and LSS is one of the major dilemmas among spine surgeons. Both types of fixation have some advantages among the other: LSS provides good implant and fracture stability, whereas SSS allows surgeons to save some segment of motion to preserve function of adjacent segments and save some operating time and blood loss $[18,19]$.

In a recent meta-analysis, Aly found no difference in terms of radiological and functional outcome, neurologic improvement, and rate of implant failure. However, these results were based on the observation of traumatic fracture in young patients and not in the osteoporotic population [20]. Moreover, according to our results, the analysis of kyphotic correction made by Assuncao Filho et al. [21] indicated poorer corrections for SSS immediately after surgery and a greater loss of this correction in the longterm follow-up. In our comparative study, we did not find a worse loss of kyphotic correction over time; in fact, after 3 months from surgery, kyphotic deformity tended to remain stable over time. This result could be associated to the use of intermediate screw at the fracture level, which improved stability of the construct [22,23], allowing surgeons to save some segments of motion and achieving maintenance of sagittal alignment similar to an LSS [24]. On the other hand, LSS still remains a valuable choice in highly unstable fractures, where a longer stabilization provides optimal stability [22].

Based on these premises, OVF should be considered as potentially highly unstable fractures, owing to poor bone quality and strength [25]. In cases with very fragmented vertebral body collapse, posterior LSS offers better clinical and radiological outcomes with lower incidence of mechanical complications [26]. However, LSS exposes patients to longer operating time and more blood loss along with a higher risk of implant infection [27].

The recent finite element analysis performed by $\mathrm{Wu}$ et al. [28] analyzed the behavior of LSS and SSS for severe thoracolumbar fracture suggested that LSS is a better choice in osteoporotic patients, similar to our results. LSS provides the greatest mechanical stiffness and can reduce further segmental collapse. However, the authors also reported some limitations to their experiment such as the analysis of a single-level injury, the assumption that the vertebral body is homogeneous, no interaction with muscle contraction and stabilization function, and data that did not take into consideration individual physiological differences [28].

Following the DGOU recommendation, an SSS is indicated for type 3 OVF, whereas an LSS is recommended for types 4 and 5. In our retrospective study, our treatment choice and DGOU recommendation were in agreement in most of cases, except for three patients with OF 4 fracture treated with SSS [14]. Two of these three patients had mechanical complications. However, in the other case, we chose to perform cement augmentation and no mechanical complication was recorded. These data were insufficient to gain any definitive conclusion, but they suggest that even in case of an inadequate treatment the use of augmentation technique could help surgeons in lowering mechanical complications, especially in OF 4 fracture that involves different shapes of fracture [16]. Nevertheless, it must be noted that SSS with augmentation did not result in expected biomechanical benefits and lengthening of dorsal instrumentation resulted in higher resistance to fatigue test and pullout in biomechanical test of cadaveric specimens [29].

To the best of our knowledge, this is the first study analyzing clinical and radiological outcomes of OVF in elderly patients; other studies in the literature were focused on whole vertebral fracture without distinction between young and elderly population.

This study has some limitations. Some are intrinsic to the study design, such as the lack of randomization and the retrospective nature. The decision of performing LSS or SSS has been entrusted to surgeon's own experience 
and types of fracture, without following DGOU recommendations, because all cases were treated before the development of the classification [16].

Moreover, only a minimum follow-up time was set with 24-48 months. Moreover, the small number of patients and the lack of a priori sample size calculation could be sources of potential bias. No systematic investigation of global spine alignment was performed. This could be considered a source of bias because misalignment of the spine and alterations of spinopelvic parameters could influence global outcomes, as described in an analysis in young and elderly patients [30,31].

\section{Conclusions}

Both treatments showed good clinical and radiological results; however, the LSS group showed better BKA correction and lower mechanical complications than the SSS group during the follow-up period.

\section{Conflict of Interest}

No potential conflict of interest relevant to this article was reported.

\section{References}

1. Wood KB, Li W, Lebl DR, Ploumis A. Management of thoracolumbar spine fractures. Spine J 2014;14:14564.

2. Johnell O, Kanis J. Epidemiology of osteoporotic fractures. Osteoporos Int 2005;16 Suppl 2:S3-7.

3. Girardo M, Rava A, Coniglio A, et al. Importance of polymethylmethacrylate augmentation in the treatment of thoracolumbar osteoporotic vertebral fractures. Minerva Ortop Traumatol 2019;70:65-9.

4. Girardo M, Rava A, Fusini F, Gargiulo G, Coniglio A, Cinnella P. Different pedicle osteosynthesis for thoracolumbar vertebral fractures in elderly patients. Eur Spine J 2018;27(Suppl 2):198-205.

5. Girardo M, Rava A, Aprato A, Masse A, Fusini F. The use of percutaneous vertebral augmentation systems in osteoporotic fractures of thoracolumbar spine. Minerva Ortop Traumatol 2019;70:86-90.

6. Girardo M, Cinnella P, Gargiulo G, Viglierchio P, Rava A, Aleotti S. Surgical treatment of osteoporotic thoraco-lumbar compressive fractures: the use of pedicle screw with augmentation PMMA. Eur Spine J 2017;26(Suppl 4):546-51.

7. Gargiulo G, Girardo M, Rava A, et al. Clinical comparison between simple laminectomy and laminectomy plus posterior instrumentation in surgical treatment of cervical myelopathy. Eur J Orthop Surg Traumatol 2019;29:975-82.

8. Chotigavanich C, Sanpakit S, Wantthanaapisith T, Thanapipatsiri S, Chotigavanich C. The surgical treatment of the osteoporotic vertebral compression fracture in the elderly patients with the spinal instrumentation. J Med Assoc Thai 2009;92 Supp15:S109-15.

9. Girardo M, Rava A, Gargiulo G, et al. Clinical and radiological union rate evaluation of type 2 odontoid fractures: a comparison between anterior screw fixation and halo vest in elderly patients. J Craniovertebr Junction Spine 2018;9:254-9.

10. Lehman RA Jr, Kang DG, Wagner SC. Management of osteoporosis in spine surgery. J Am Acad Orthop Surg 2015;23:253-63.

11. Kohno M, Iwamura Y, Inasaka R, et al. Surgical intervention for osteoporotic vertebral burst fractures in middle-low lumbar spine with special reference to postoperative complications affecting surgical outcomes. Neurol Med Chir (Tokyo) 2019;59:98-105.

12. Basaran R, Efendioglu M, Kaksi M, Celik T, Mutlu I, Ucar M. Finite element analysis of short- versus longsegment posterior fixation for thoracolumbar burst fracture. World Neurosurg 2019;128:e1109-17.

13. Ahsan MK, Mamun AA, Zahangiri Z, et al. Shortsegment versus long-segment stabilization for unstable thoracolumbar junction burst fractures. Mymensingh Med J 2017;26:762-74.

14. Schnake KJ, Blattert TR, Hahn P, et al. Classification of osteoporotic thoracolumbar spine fractures: recommendations of the spine section of the German Society for Orthopaedics and Trauma (DGOU). Global Spine J 2018;8(2 Suppl):46S-49S.

15. Rollinghoff M, Zarghooni K, Schluter-Brust K, et al. Indications and contraindications for vertebroplasty and kyphoplasty. Arch Orthop Trauma Surg 2010;130:765-74.

16. Blattert TR, Schnake KJ, Gonschorek O, et al. Nonsurgical and surgical management of osteoporotic vertebral body fractures: recommendations of the spine section of the German Society for Orthopaedics and Trauma (DGOU). Global Spine J 2018;8(2 
Suppl):50S-55S.

17. Schnake KJ, Hahn P, Franck A, et al. Development of a classification system (OF-classification) and a score for therapeutic decision-making (OF-score) for osteoporotic thoracolumbar fractures. Glob Spine J 2015;5(1_Suppl):s-0035.

18. Schulze M, Gehweiler D, Riesenbeck O, et al. Biomechanical characteristics of pedicle screws in osteoporotic vertebrae-comparing a new cadaver corpectomy model and pure pull-out testing. J Orthop Res 2017;35:167-74.

19. Canbek U, Karapınar L, Imerci A, Akgun U, Kumbaracı M, Incesu M. Posterior fixation of thoracolumbar burst fractures: is it possible to protect one segment in the lumbar region? Eur J Orthop Surg Traumatol 2014;24:459-65.

20. Aly TA. Short segment versus long segment pedicle screws fixation in management of thoracolumbar burst fractures: meta-analysis. Asian Spine J 2017;11:150-60.

21. Assuncao Filho CA, Simoes FC, Prado GO. Thoracolumbar burst fractures, short X long fixation: a metaanalysis. Coluna/Columna 2016;15:78-84.

22. Baaj AA, Reyes PM, Yaqoobi AS, et al. Biomechanical advantage of the index-level pedicle screw in unstable thoracolumbar junction fractures. J Neurosurg Spine 2011;14:192-7.

23. Sallam AM, Ghany WA, Ali AK, et al. Short-segment posterior fixation with index level screws versus long-segment posterior fixation for thoracolumbar spine fracture: angle of correction and pain. Egypt J Neurosurg 2018;33:11.

24. Dobran M, Nasi D, Brunozzi D, et al. Treatment of unstable thoracolumbar junction fractures: short- segment pedicle fixation with inclusion of the fracture level versus long-segment instrumentation. Acta Neurochir (Wien) 2016;158:1883-9.

25. Ferguson SJ, Steffen T. Biomechanics of the aging spine. Eur Spine J 2003;12 Suppl 2(Suppl 2):S97-S103.

26. Waqar M, van-Popta D, Barone DG, Bhojak M, Pillay R, Sarsam Z. Short versus long-segment posterior fixation in the treatment of thoracolumbar junction fractures: a comparison of outcomes. Br J Neurosurg 2017;31:54-7.

27. Li J, Liu L. Comparison of short-segment versus long-segment fixation for the treatment of thoracolumbar burst fracture: a meta-analysis. Int J Clin Exp Med 2017;10:1750-62.

28. Wu Y, Chen CH, Tsuang FY, Lin YC, Chiang CJ, Kuo YJ. The stability of long-segment and short-segment fixation for treating severe burst fractures at the thoracolumbar junction in osteoporotic bone: a finite element analysis. PLoS One 2019;14:e0211676.

29. Weiser L, Dreimann M, Huber G, et al. Cement augmentation versus extended dorsal instrumentation in the treatment of osteoporotic vertebral fractures: a biomechanical comparison. Bone Joint J 2016;98B:1099-105.

30. Langella F, Villafane JH, Lafage V, et al. Xiphopubic angle (XPA) correlates with patient's reported outcomes in a population of adult spinal deformity: results from a multi-center cohort study. Eur Spine J 2018;27:670-7.

31. Berjano P, Langella F, Ismael MF, Damilano M, Scopetta S, Lamartina C. Successful correction of sagittal imbalance can be calculated on the basis of pelvic incidence and age. Eur Spine J 2014;23 Suppl 6:587-96. 